\title{
Interférences
}

Ars scribendi

\section{Vies parallèles dans le récit livien : Hannibal et Scipion l'Africain}

\section{Bernard Mineo}

\section{(2) OpenEdition \\ 1 Journals}

Édition électronique

URL : http://journals.openedition.org/interferences/911

DOI : 10.4000/interferences.911

ISSN : $1777-5485$

Éditeur

HiSoMA - Histoire et sources des Mondes antiques

Édition imprimée

Date de publication : 1 janvier 2009

ISSN : 1777-5485

\section{Référence électronique}

Bernard Mineo, «Vies parallèles dans le récit livien : Hannibal et Scipion l'Africain », Interférences [En ligne], 5 | 2009, mis en ligne le 10 juillet 2014, consulté le 15 septembre 2020. URL : http://

journals.openedition.org/interferences/911; DOI : https://doi.org/10.4000/interferences.911

Ce document a été généré automatiquement le 15 septembre 2020.

Tous droits réservés 


\title{
Vies parallèles dans le récit livien : Hannibal et Scipion l'Africain
}

\author{
Bernard Mineo
}

1 Il pourrait paraître a priori invraisemblable que Tite-Live eût jamais pu concevoir le projet de mettre en parallèle les motifs biographiques de la vie d'Hannibal, l'ennemi mortel de Rome, avec ceux de celui qui en fut le sauveur, Scipion l'Africain. En quoi la vie de celui qui incarnait le génie carthaginois, vu par les Romains, dans ses pires excès (la mala fides, la superbia, la sceuitia) pouvait-elle être le miroir de celui qui portait en lui au plus haut degré certaines des vertus sur lesquelles reposait l'idéal livien de la romanité (notamment la fides, la moderatio, la liberalitas, la clementia, la pietas)? La tentation de comparer tout au moins le génie militaire des deux plus grands capitaines de la deuxième guerre punique s'est pourtant fait sentir très tôt. On devine que Polybe fut l'un des premiers à s'engager dans cette voie. Au reste, l'intérêt marqué pour les biographies, qu'un Varron ou un Cornelius Nepos ${ }^{1}$ ont pu contribuer à populariser au $\mathrm{I}^{\mathrm{er}}$ siècle av. J.-C., fait qu'on ne saurait s'étonner de la présence de motifs et de structures propres à ce type de discours chez Tite-Live. De fait, celui-ci, dès le début de son récit de la Guerre d'Hannibal, semble guidé par le souci d'établir des analogies entre la carrière militaire et politique du général carthaginois et celle de Scipion. Les quatre premiers chapitres à teneur biographique du livre XXI, qui permettent d'introduire le fils d'Hamilcar, sont ainsi le point de départ et comme la clé de voûte d'une entreprise qui ne s'achève qu'avec l'évocation de la mort d'Hannibal, et la comparaison que l'historien établit à l'occasion entre la vie de ce général et celles de Scipion et de Philopœmen.

2 Le propos de cet exposé sera donc d'essayer d'illustrer la réalité de ce projet de vies parallèles et d'en éclairer la raison d'être. À cette fin, il conviendra d'examiner tour à tour les motifs qui permettent à l'auteur de souligner les analogies entre les deux héros, de dégager ensuite les structures narratives porteuses de ces parallélismes biographiques ainsi que les procédés rhétoriques les mettant en valeur (amplification, dispositio) ; il importera, enfin, de faire apparaître les éléments de différenciation entre les deux vies susceptibles d'éclairer le sens de la construction livienne. 


\section{Motifs parallèles}

Le premier motif parallèle entre les deux héros tient au fait que leur autorité militaire paraisse en partie déterminée par leur appartenance à une dynastie de grands généraux. Comme si l'on eût affaire à de véritables princes, leur politique porte l'empreinte profonde de leur personnalité et de leurs passions, menaçant parfois l'équilibre des institutions.

4 Hannibal est ainsi présenté avant tout, dans le prologue biographique du livre XXI, comme le digne fils de son père, dont il reprend le projet de vengeance contre Rome. Hannibal possède en lui en effet l'immense orgueil d'Hamilcar, blessé par la perte de la Sicile et de la Sardaigne, à la suite de quoi il avait cherché à développer l'Empire carthaginois en Espagne, et à préparer une guerre de revanche (Liv., XXI, 1, 5;2,1-2). C'est au moment où Hamilcar se prépare du reste à faire passer son armée en Espagne que se situe le fameux passage évoquant le serment par lequel Hannibal aurait juré d'être l'ennemi du peuple romain dès qu'il le pourrait, alors qu'il n'était âgé que de neuf ans (XXI, 1, 4) ${ }^{2}$. Dans cette optique, la deuxième guerre punique n'apparait plus comme l'affaire de la cité, dictée par des impératifs rationnels, mais comme la satisfaction de passions particulières, la haine et l'ambition des Barcides, avides de revanche et de gloire, utilisant les ressources d'une Espagne sur laquelle ils exercent une véritable souveraineté pour conduire une politique hostile à Rome. Cette thèse, réfutée par Polybe, remonte à Fabius Pictor, qui semble avoir ainsi cherché à illustrer la sagesse de la politique de son cousin, Fabius Maximus, hostile à la ligne stratégique des Scipions, et serait allé jusqu'à affirmer qu'Hannibal n'avait pas l'aval de Carthage ${ }^{3}$.

Si Tite-Live, qui refuse de dédouaner le sénat carthaginois ${ }^{4}$, marque son désaccord avec ce dernier point de vue, il conserve pour le reste la même interprétation des événements que Fabius Pictor, comme le fait notamment apparaître son évocation des circonstances qui ont favorisé l'ascension d'Hasdrubal, le successeur d'Hamilcar à la tête de l'armée d'Espagne. Celui-ci doit donc son commandement à «la puissance de la faction des Barca, dont l'influence sur les soldats et la plèbe était supérieure à la moyenne, et non, certes, en raison de la volonté des grands » (Liv., XXI, 2, 4).

Ces liens personnels avec l'élément populaire de la cité et l'armée seraient donc allés jusqu'à encourager un comportement monarchique qui, déjà dans le passé, aurait amené Hamilcar et Hasdrubal à gouverner l'Espagne sans tenir compte du sénat, après l'échec de la tentative de renverser la constitution de la cité punique à leur profit. La crainte de voir Hannibal développer les mêmes appétits royaux que ses deux prédécesseurs est au reste formulée très clairement par Hannon le Grand dans le discours (absent chez Polybe) que celui-ci prononce devant le sénat de Carthage pour s'opposer à l'envoi d'Hannibal en Espagne malgré son très jeune âge (uixdum puberem), ainsi que le réclame Hasdrubal : «Craignons-nous que le fils d'un Hamilcar ne voie trop tard les pouvoirs démesurés et l'éclat de la royauté exercée par son père (regni paterni speciem) et que nous ne soyons pas assez tôt les esclaves du fils d'un roi, au gendre duquel nos armées ont été laissées comme des biens héréditaires? Pour moi, je suis d'avis de garder ce jeune homme dans sa patrie, soumis aux lois, soumis aux magistrats, et de lui apprendre à vivre avec des droits qui ne soient pas supérieurs à ceux d'autrui, de peur qu'un jour cette petite flamme ne fasse surgir un incendie» (Liv., XXI, 3, 5, trad. CUF). Point de vue qui ne sera suivi que par quelques sénateurs, les meilleurs, 
écrit Tite-Live $(4,1)$. D'un côté donc le souci du primat républicain, de l'autre, le risque de voir le jeune Hannibal hériter des pouvoirs démesurés de son père (imperia immodica) et menacer d'exercer une sorte de royauté au moins en Espagne.

7 Tite-Live ne manque pas au reste de souligner l'espèce de continuité dynastique qui permet à Hannibal de s'imposer aisément à la tête de l'armée carthaginoise, dès son arrivée en Espagne, ce que facilite sa forte ressemblance physique et morale avec son père :

Envoyé en Espagne, Hannibal attira sur lui, dès son arrivée, l'attention de toute l'armée; les vieux soldats croyaient qu'Hamilcar jeune leur avait été rendu; ils voyaient la même énergie dans le visage, la même vivacité dans les yeux, le même air et les mêmes traits. Il fit ensuite rapidement en sorte que sa ressemblance avec son père l'aidât le moins possible à gagner les sympathies 5 .

8 Même si l'autorité d'Hannibal tient pour une grande part à ses qualités personnelles, elle doit donc également beaucoup au lien affectif particulier qui lie la troupe au représentant d'une lignée de chefs eux-mêmes charismatiques, dans une logique quasidynastique où la loyauté envers le général précède la fides à l'endroit de la cité. Les fondements de ce pouvoir sont donc essentiellement populaires, militaires, et font aisément fi de l'autorité sénatoriale et de l'intérêt de l'État.

Or, on est frappé de constater comment le récit livien s'évertue sur ce point à établir des parallélismes entre les deux grandes figures de la deuxième guerre punique, ce qu'encourageait historiquement le fait que la geste de Scipion l'Africain commençait véritablement par sa prise de commandement de l'armée d'Espagne où le jeune homme succédait à son père et à son oncle, de même que son adversaire carthaginois avait pris la suite d'Hamilcar puis d'Hasdrubal.

10 L'ombre du père et de l'oncle est de fait présente dès les premières lignes qui introduisent le passage consacré à l'imperium d'Espagne ${ }^{6}$. Mais l'idée que le jeune Scipion opère une succession quasi-dynastique à la tête de l'armée romaine, à l'instar d'Hannibal, est surtout perceptible à travers la façon dont l'auteur amplifie le thème de la ressemblance physique et morale du jeune homme avec ses deux parents, dans un discours au style direct où le personnage revendique lui-même l'héritage d'une fides particulière qui lierait la troupe aux Scipions :

Montrez seulement, soldats, votre fidélité aux Scipions, au rejeton si j'ose dire de vos généraux, qui repart de l'arbre qui a été abattu [...] Bientôt, je vous le garantis, comme vous trouvez dans mon regard, dans mon expression et dans mes traits, une ressemblance avec mon père et avec mon oncle, vous trouverez aussi en moi la réplique, le reflet de leur intelligence, [...] si bien que chacun dira qu'il voit revivre ou plutôt renaître le général Scipion ${ }^{7}$...

11 Il n'est pas question en revanche de comportement non républicain des Cornelii ou de Scipion à la tête de leurs armées : tous restent de fidèles généraux soumis au Sénat de Rome, à la différence des Barcides. Cependant, le futur Africain esquissera bientôt une nouvelle attitude politique lorsqu'il cherchera à imposer son expédition africaine au Sénat récalcitrant et que, dans un passage sur lequel il faudra revenir, il menacera de s'appuyer sur les comices en cas d'obstination des Pères ${ }^{8}$, au point que Fabius Maximus pourra l'accuser en 205, devant le Sénat, de se comporter regio more, à la façon des rois.

12 Un autre thème, introduit par la mini-biographie qui sert d'ouverture au livre XXI, insiste quant à lui sur la jeunesse excessive du futur général punique à différents moments clés de sa carrière, et sur son exceptionnelle popularité. 
13 La jeunesse d'Hannibal sera ainsi montrée du doigt quand il succédera à Hasdrubal en Espagne, mais sa désignation s'accompagne d'une autre caractéristique importante, à savoir le consensus des soldats, qui lui décernent le titre d'imperator, et celui de la plèbe qui se prononce à l'unanimité sur son nom :

Il n'y eut pas d'hésitation pour savoir qui succéderait à Hasdrubal ; le premier choix des soldats qui avaient aussitôt conduit le jeune Hannibal au prétoire et lui avaient donné le titre de général en chef au milieu d'immenses clameurs et dans un contentement unanime fut suivi d'une égale approbation de la plèbe ${ }^{9}$.

L'ascension du personnage est donc portée par une popularité hors du commun.

L'étonnante jeunesse de Scipion et son immense popularité seront de la même façon mises en valeur dans le récit livien. Plusieurs éléments interviendront ainsi dans la dramatisation de son élection à l'édilité : le fait que Scipion n'avait pas encore l'âge requis (il n'avait que 22 ou 23 ans), l'opposition des tribuns de la plèbe et finalement le consensus autour de son nom ${ }^{10}$.

15 L'épisode du vote de l'imperium d'Espagne concourait lui aussi à souligner ces mêmes caractéristiques qui font de Scipion un général hors du commun :

On avait d'abord attendu que les personnages qui se croyaient dignes d'un si grand commandement présentent leur nom [...] C'est alors que P. Cornelius, le fils du Publius tombé en Espagne, un jeune homme âgé d'à peine vingt-quatre ans, déclara sa candidature et s'installa à une place élevée d'où l'on pouvait l'apercevoir. Tous les regards s'étant tournés vers lui, tout aussitôt des acclamations enthousiastes présagèrent un commandement heureux et favorable (clamore ac fauore ominati extemplo sunt felix faustumque imperium). Invités ensuite à voter, c'est de façon unanime que non seulement toutes les centuries, mais aussi tous les individus votèrent en faveur du commandement de Scipion en Espagne ${ }^{11}$.

16 On ne manquera pas de relever ici le caractère paradoxal de ces remarques, en raison de l'anachronisme de cette référence à un âge légal avant le vote des premières leges Annales. Il fallut, en effet, attendre la Lex Villia Annalis de 180 pour que soit précisé l'âge requis pour exercer les magistratures curules. Une réalité que Tite-Live n'ignore pas, puisque c'est lui-même qui nous offrira au livre XL quelques précieuses indications sur cette mesure ${ }^{12}$. On comprend que le dessein de Tite-Live ici est de faire de la jeunesse de Scipion et d'Hannibal, et du consensus que tous deux suscitent, des indices de leur destin exceptionnel de condottieri, et de révéler l'ambition hors du commun de ces deux personnages.

17 On pourrait relever d'autres caractéristiques similaires chez ces deux hommes, en particulier le charisme qui les rend chers à leurs soldats (Scipion est lui aussi proclamé imperator par ses troupes ${ }^{13}$ ) et leur permet également de se concilier, dans le cas de Scipion, les princes et les populations étrangères ${ }^{14}$, comme savait le faire Hasdrubal, le prédécesseur du vainqueur de Cannes ${ }^{15}$. Leur capacité à se faire entendre et obéir découle encore de leur commune habilité oratoire, reposant sur une audace morale particulière, qui leur inspire des mises en scène spectaculaires : à l'absence de scrupule prêtée à Hannibal (Liv., XXI, 4, 5-10) correspond de fait le sens de la manipulation religieuse chez Scipion, ce qui ne manque pas de susciter des remarques aigres-douces de Tite-Live ${ }^{16}$. Cette tradition se trouvait déjà chez Polybe qui cependant, à l'inverse de l'historien padouan, trouvait très habile cette façon de faire valoir ses vues ${ }^{17}$.

18 Enfin l'ambition d'Hannibal n'a d'égale que celle de Scipion, lorsque celui-ci professe son ambition de terminer la guerre (XXVIII, 40,1), de surpasser la gloire du Cunctator 
(XXVIII, 43) ou lorsque l'auteur évoque ses tourments à l'idée de laisser à un éventuel successeur la gloire de terminer le conflit (XXX, 36).

On voit donc bien qu'en prêtant à Scipion quelques-unes des caractéristiques biographiques sur lesquelles est construit son personnage d'Hannibal, Tite-Live aura voulu éclairer la fonction narrative de l'Africain, qui est d'être la première figure romaine à laquelle la jeunesse, le charisme et l'ambition confèrent une dimension héroïque, sur le modèle hellénistique, dont la référence par excellence est celle d'Alexandre le Grand.

\section{Destins parallèles et entrecroisés : structures narratives, mise en valeur rhétorique}

Plus encore cependant que les motifs parallèles, ce sont les structures narratives ellesmêmes qui disent la centralité du projet livien d'éclairer les deux vies l'une par l'autre.

On remarquera tout d'abord qu'Hannibal et Scipion sont les deux seuls personnages de la troisième décade faisant l'objet d'un excursus biographique permettant de définir leurs principales qualités. Ces développements interviennent l'un et l'autre lorsque les deux généraux se trouvent engagés de façon déterminante dans leur trajectoire, à savoir au moment de leur envoi ou de leur arrivée en Espagne (Hannibal : XXI, 1-4; Scipion : XXVI, 19, 3-9).

22 Mais la réalité de ces vies parallèles liviennes est surtout perceptible dans la construction en miroir des deux débats sénatoriaux qui déterminent le destin des deux généraux ennemis : celui où intervient Hannon, dans l'introduction biographique du livre XXI, où l'on voit le personnage s'opposer au projet d'envoyer le jeune Hannibal en Espagne avant qu'il ait terminé sa formation civique à Carthage et le long débat dramatisé qui oppose Fabius Cunctator et Scipion sur l'opportunité d'une expédition en Afrique en 205. La mise en miroir de ces deux passages constitue sans doute la construction la plus originale de Tite-Live, puisqu'on ne trouve aucune trace de l'intervention d'Hannon à quelque moment que ce soit chez Polybe ou de façon générale dans la tradition pré-livienne. Nous nous attarderons d'autant plus longuement sur ce parallélisme que celui-ci offre sans doute la clé de lecture du rapprochement voulu par Tite-Live entre les deux héros.

De fait, la symétrie des circonstances de ces deux débats est telle qu'il paraît invraisemblable de l'imputer au hasard. Tous deux se déroulent dans une enceinte sénatoriale, carthaginoise puis romaine, et ont finalement le même objet: donner l'aval à une disposition qui sera historiquement pour les deux nations le point de départ d'une périlleuse aventure impériale. Dans le premier cas, les sénateurs ouvrent la voie, sans le savoir, à la guerre d'Hannibal, qui s'avérera un désastre pour Carthage et un épisode supplémentaire de l'impérialisme carthaginois outre-mer, déjà marqué par l'échec en Sicile, en Sardaigne et en Corse ; pour Rome, il s'agit d'un premier pas, si l'on excepte la tragique tentative de Régulus, vers une expansion méditerranéenne qui lui donnera l'empire du monde et l'éloignera définitivement de son berceau italien. En d'autres termes, Rome emboîte le pas à Carthage dans une voie et une logique dont la cité punique a déjà pu sentir, dans le passé, les effets pernicieux.

Surtout, Hannon comme Fabius, dans les deux débats, font l'un et l'autre figures de sages expérimentés. Le Cunctator est proche de sa fin (Tite-Live évoquera sa mort 
en 203 ${ }^{18}$, et Hannon le Grand suffisamment âgé pour avoir déjà commandé les forces carthaginoises à la fin de la première guerre punique, et lors de la guerre des mercenaires ${ }^{19}$.

En outre, de même que Tite-live évoque le soutien que les plus sages ont apporté à Hannon (XXI, 4), il ne manque pas non plus de préciser que les sénateurs d'un certain âge n'appréciaient guère la fougue de Scipion (XXVIII, 43) ni les menaces de ce dernier de s'appuyer sur les comices pour forcer la décision du Sénat (XXVIII, 40). L'opposition anachronique optimates/populares, nette du côté d'Hannibal, est seulement esquissée pour Scipion.

C'est en tout cas cette expérience et leur âge fort avancé qui permettent à Hannon et à Fabius de figurer dans le récit comme de véritables uates, capables d'entrevoir l'avenir. Ainsi, fort de sa sagesse, lors de la séance au Sénat où les Carthaginois choisiront la guerre, Hannon peut-il énoncer les raisons juridiques et religieuses qui condamnent sa cité à la défaite (XXI, 10). Tite-Live souligne aussi l'isolement du personnage parmi les sénateurs, alors que Polybe se contente de souligner que l'on confia au plus qualifié d'entre eux de présenter les arguments de ses collègues; celui-ci aurait alors dénié toute légitimité aux prétentions romaines, à l'inverse de ce que fait Hannon chez Tite-Live (Pol., III, 20, 10-21, 1-5).

De plus, le procès que Fabius fait à Scipion est exactement le même que celui qu'intente Hannon à Hannibal au livre XXI : on se rappelle que le vieux sénateur y exprimait sa crainte qu'en envoyant trop tôt le fils d'Hamilcar à l'armée, celui-ci n'eût pas le temps d'apprendre à se soumettre aux lois et aux magistrats, et qu'il ne se comportât à l'avenir comme un roi $(\mathrm{XXI}, 3)^{20}$. De la même façon, le Cunctator affirme à la fin de son intervention que seul le souci de sa gloire a inspiré à Scipion son dessein de passer en Afrique, tandis qu'il dénonce au passage l'attitude orgueilleuse et monarchique de son adversaire :

Tu te soucies plus de ta carrière que de l'intérêt de l'État; et c'est pour les mêmes raisons que tu as quitté ta province et ton armée, toi, le général du peuple romain, sans que t'y autorise une loi ou un senatus-consulte et que tu as confié le sort de l'État et la puissance de Rome à deux navires. Mon avis, c'est que Publius Cornélius Scipion a été élu consul pour l'état et pour nous, et non à titre privé et pour son intérêt personnel ; que les armées ont été enrôlées pour défendre Rome et l'Italie, non pour que des consuls, à la façon de rois agissant comme des tyrans (regio more per superbiam), les emmènent où bon leur semble ${ }^{21}$.

Or, cette mise en garde de Fabius, loin d'être l'expression d'un simple affrontement de circonstances, est au contraire centrale si l'on veut bien comprendre les enjeux historiques que Tite-Live veut faire apparaître à ce point de son récit.

L'historien sait en effet que le lecteur cultivé ne manquera pas de saisir la signification particulière de cette mise en scène et qu'il saura faire le rapprochement avec les discours que prononcent, chez Thucydide, Nicias et Alcibiade devant le peuple athénien sur l'opportunité d'une expédition en Sicile contre Syracuse ${ }^{22}$. De fait, les analogies entre les deux textes sont précises et nombreuses.

On remarquera notamment que, chez Thucydide comme chez Tite-Live, la ligne de partage des opinions recoupe une opposition entre générations. Ainsi, Nicias rappelle que la jeunesse a répondu à Alcibiade tandis que lui-même s'est tourné vers des hommes âgés ${ }^{23}$. De la même façon, on l'a vu, Fabius évoque sa vieillesse et dit combien il est fatigué de la vie pour démontrer l'absence de passion personnelle derrière sa prise de position. Alcibiade, pour sa part, est convaincu que sa jeunesse est un atout ${ }^{24}$, 
tandis que la fascination qu'exerce la jeunesse de Scipion au moment où il accède à ses premières responsabilités militaires et politiques est régulièrement rappelée dans notre texte ${ }^{25}$. Pour autant, la fonction narrative des deux couples de personnages n'est pas strictement identique : alors que Thucydide n'est préoccupé que par l'analyse des caractères, Nicias symbolisant simplement l'homme d'expérience, et Alcibiade la folle impétuosité de la jeunesse, Tite-Live utilise ce point de départ fondé sur les ressorts psychologiques d'un conflit de générations pour investir ses deux héros d'une fonction plus large qui en fait les représentants de deux logiques historiques différentes, Fabius étant l'homme du passé et Scipion celui du futur.

31 L'analogie entre les personnages se retrouve encore dans la façon dont les deux auteurs les caractérisent : Alcibiade se comporte avec la plus extrême indépendance d'esprit ${ }^{26}$, de même que Scipion se désolidarise des comportements traditionnels en vivant à la grecque, suscitant de la part de ses adversaires au Sénat suffisamment d'inquiétude pour qu'une commission soit dépêchée en Sicile pour enquêter sur les mœurs du futur Africain ${ }^{27}$.

se retrouvent encore au fond même des débats. Nicias et Fabius, reprochent en effet à leurs adversaires de rechercher leur intérêt personnel et non celui de l'État ${ }^{28}$; tout comme Fabius refuse l'idée d'une expédition hors d'Italie, Nicias dénonce un projet qui conduira les Athéniens à sortir de leurs frontières actuelles en cédant à un «appétit malsain des choses lointaines ${ }^{29} »$. Enfin, le soupçon de tyrannie que son comportement

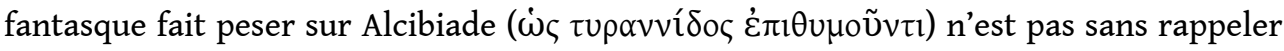
l'orgueil tyrannique et royal qui, selon Fabius, inspirerait à Scipion son projet de conduire les forces romaines où bon lui semble ${ }^{30}$.

Le lien entre les deux débats était au demeurant d'autant plus difficile à ne pas établir que Tite-Live, par l'intermédiaire de Fabius, invitait explicitement son lecteur à se rappeler les suites désastreuses de l'expédition de Sicile, et à comparer Scipion à Alcibiade ${ }^{31}$.

34 Bien entendu, l'histoire d'Athènes n'était pas celle de Rome: au lieu de la défaite de l'Assinaros, les Romains allaient connaître la victoire de Zama. Mais c'est sur l'orientation de la nouvelle tendance historique induite par l'expédition africaine de Scipion que le lecteur de l'Ab Vrbe Condita était invité à méditer, et sur les leçons d'une histoire qui avait démontré les périls d'un impérialisme démesuré.

Cette nouvelle évolution s'intègre en réalité dans une conception de l'histoire qui structure l'ensemble de l'œuvre livienne, et qui fait se succéder plusieurs cycles historiques d'égale durée: le deuxième a débuté par la refondation de Rome par Camille et conduit l'Vrbs à son apogée lors de la deuxième guerre punique. Le tournant, le cardo temporel, intervient autour de 207, ainsi que j'ai essayé de le montrer dans un ouvrage récemment publié ${ }^{32}$. À partir de la bataille du Métaure, où le metus hostilis s'est considérablement affaibli, les destins de l'Vrbs commencent en effet à suivre un cours nouveau: n'étant plus retenus par le péril mortel encouru par la cité, les comportements se modifient progressivement au sein de la classe dirigeante, et l'on voit bientôt des chefs, exaltés par la puissance grandissante de la cité, rivaliser en gloire les uns avec les autres; dans le même temps, le contact avec l'Orient hellénistique favorise la luxuria et l'auaritia, autant de vices qui conduiront petit à petit les Romains à la guerre civile. construction en miroir des interventions au Sénat d'Hannon et de Fabius Cunctator est 
donc loin d'être anodin; il confirme ce que laissait apparaître déjà l'étude des motifs biographiques mis en valeur au livre XXI, à savoir le dessein de l'historien d'intégrer le personnage de Scipion à l'intérieur d'une typologie à laquelle Hannibal appartient également, celle des grands généraux hellénistiques dont Scipion est le prototype à Rome, une lointaine préfiguration des Marius, Sylla, Pompée et César qui conduiront la République à sa perte.

Ce parallélisme entre les deux vies et finalement entre l'histoire des deux cités est du reste suffisamment fondamental pour inspirer la structure en boucle de la troisième décade : cette dernière s'ouvre, de fait, sur la présentation biographique d'Hannibal, et notamment sur le débat où Hannon dénonce le danger en puissance que représente ce dernier. Elle se referme, en revanche, sur l'image du triomphe de Scipion au livre XXX, dont les dernières lignes soulignent le fait que celui-ci fut le premier général passé à la postérité sous le nom du peuple qu'il avait vaincu, comme on devait le faire du temps de Sylla qui se faisait appeler Heureux, ou de Pompée le Grand (XXX, 45). La roue du temps a tourné, et le danger potentiel que fait courir le comportement royal des généraux ambitieux qui n'hésitent pas, dans leur désir de se couvrir de gloire, à conduire leurs armées loin de leur patrie, est passé de Carthage à Rome ${ }^{33}$.

L'amplification du thème de l'ingratitude et de l'hostilité dont les deux héros furent l'objet de la part de leurs concitoyens après leurs exploits constitue un autre moyen pour l'auteur de rendre visible le parallélisme des deux vies. C'est notamment le cas dans l'épisode situé en 196, où l'on voit Hannibal, suffète de Carthage, se heurter à l' inuidia des riches dont il dénonce les injustices (XXXIII, 46). L'appui que Scipion apporte à son ancien adversaire en désapprouvant les attaques insidieuses dont il est l'objet (XXXIII, 47) souligne sans doute déjà la communauté de destin entre les deux personnages, puisque Scipion doit lui aussi être victime de l'inuidia que suscite sa gloire : cette dernière est évoquée dès les élections pour l'année 192 (XXXV, 10,5), mais elle éclate à propos du récit dramatisé du procès des Scipions. S'inspirant apparemment de Valerius Antias, Tite-Live rapporte longuement au style indirect les réactions provoquées par les accusations des tribuns contre Scipion. Le parallèle établi avec Hannibal y est explicite :

Presque en même temps, les deux plus grandes villes de la terre s'étaient montrées ingrates à l'égard de leur héros. Mais c'était pire dans le cas de Rome : si Carthage, vaincue, condamnait Hannibal à l'exil à cause de sa défaite, Rome victorieuse exilait l'Africain malgré sa victoire ${ }^{34}$.

Le même passage, qui présente aussi l'autre point de vue sur le procès, confirme la nature de la problématique fondant le parallèle entre les deux personnages :

Selon d'autres, aucun citoyen ne devait s'élever au-dessus des autres au point d'échapper à l'action de la justice [...] Ceux qui ne pouvaient se résigner à l'égalité devant la loi méritaient qu'on les poursuive ${ }^{35}$.

Un peu plus loin (XXXVIII, 51, 3-4), on accuse Publius d'avoir suivi son frère Lucius en Asie :

pour montrer [...] que la grandeur de Rome reposait entièrement sur lui, que la première puissance du monde s'abritait à l'ombre de Scipion et qu'un signe de sa part remplaçait des décrets du Sénat et des suffrages populaires.

39 Tite-Live profite donc de cet épisode pour amplifier l'enjeu contenu dans les deux débats sénatoriaux liant les biographies des deux généraux rivaux, à savoir le péril pour la République que font naître des généraux trop entreprenants et avides de gloire. La dispositio du récit permet au reste d'insister sur la perspective historique du procès, puisque celui-ci intervient de façon très révélatrice après le très long passage consacré 
aux accusations portées contre le consul Cn. Manlius Vulso (XXXVIII, 45-50), lequel, avide d'obtenir un triomphe, a conduit la guerre contre les Gallo-Grecs sans l'aval du Sénat ni du peuple romain. Les risques d'un comportement regio more commencent donc à se concrétiser, illustrant les progrès de la tendance historique inaugurée par Scipion; le fait d'évoquer le procès de celui-ci juste après cet épisode, en rappelant en même temps l'inquiétude de ceux qui craignent de voir s'imposer des chefs puissants au-dessus des lois, confirme la fonction historique de l'Africain, qui, sans s'être luimême rendu coupable de vouloir bafouer les lois, a rendu ce comportement possible par sa victoire africaine et l'indépendance de ses initiatives.

L'évocation de la mort d'Hannibal est encore l'occasion d'une comparaison récapitulative entre la vie du Carthaginois et celles de Scipion et de Philopœmen, comme on pourrait en trouver dans les Vies de Plutarque (XXXIX, 52, 7-9). Polybe (XXIII, 12-14), dans un texte qui nous est parvenu dans un état fragmentaire, compare lui aussi les trois personnages au même moment du récit. Il procède surtout à un rappel de leurs exploits respectifs, pour "inciter aux belles actions les générations futures ", et souligne la fragilité de la fortune. Tite-Live insiste quant à lui sur les conditions de leur mort, sans rapport avec la gloire de ces personnages de leur vivant, et met ainsi en relief l'ingratitude dont Hannibal et Scipion furent les victimes. Le fait d'introduire ici une comparaison semble se rattacher au synchronisme contesté par Tite-Live concernant leur mort, que Polybe et Rutilius Rufus auraient tous deux située en 183 (XXXIX, 52, 1). Ce qui peut laisser penser que l'historien de Mégalopolis avait peut-être déjà établi des parallèles entre les deux vies ${ }^{36}$, un peu comme le pratiquait déjà Xénophon dans l'Anabase, lorsque celui-ci comparait les généraux. Partant de cette pratique déjà ancienne, Tite-Live aura donné une portée plus profonde à ces vies parallèles, en leur conférant la fonction de symboliser la nature de l'évolution naturelle des deux cités.

\section{Les parallélismes différenciateurs}

41 Cependant, la logique historique que suit Rome depuis la bataille du Métaure et le débarquement en Afrique sous les auspices de Scipion ne s'affirmera que progressivement, conformément aux modalités évoquées par Tite-Live dans sa préface ${ }^{37}$. Pour lors, la menace n'est que virtuelle, même si un Fabius et un Caton se montrent capables de la percevoir dans le présent. Il faudra encore beaucoup de temps avant que la tendance historique ne produise tous ses effets. Car si Rome est au tournant de son histoire, elle n'en est pas moins à son apogée politique et morale, et Scipion, son dux fatalis, qui lui ouvre une voie nouvelle, n'en est pas moins porteur des vertus de la Rome passée. D'où l'ambiguïté du personnage et la nécessité d'introduire également dans le récit des parallélismes différenciateurs, destinés à faire apparaître le décalage dialectique entre un condottiere tel Hannibal, dont la cité approche le terme de son histoire, et un Scipion, capable encore d'incarner les valeurs et les vertus qui ont conduit Rome vers son sommet historique.

42 Le premier parallèle différenciateur tient au refus de Scipion d'exercer réellement un regnum. Si ses succès, son charisme lui en ouvrent la possibilité, et en inspirent le soupçon à ses adversaires politiques, Tite-Live met à maintes reprises en valeur dans le texte le refus constant de ce dernier de céder à cette tentation. 

effectivement de son vivant contre ces accusations d'aspirer au regnum. Sa jeunesse,
son invincibilité, son audace, ne pouvaient que nourrir des soupçons parmi une aristocratie romaine bien au fait des réalités du monde grec et du prestige de la monarchie hellénistique. Il est bien possible que Fabius Pictor ait été le premier à se faire l'écho des inquiétudes que la popularité de l'Africain inspirait à son célèbre cousin, Fabius Cunctator; les éloges des poètes comme Ennius, l'influence de l'évhémérisme à cette époque, qui promettait aux grands hommes l'immortalité, n'étaient pas sans contribuer à nourrir cette méfiance. On sait que Caton fut un de ceux qui marquèrent le plus ostensiblement leur hostilité à Scipion dont la dignitas lui paraissait une menace pour l'équilibre des institutions. L'Africain dut se défendre régulièrement contre ces accusations, refusant qu'on l'élise consul à vie et dictateur, qu'on lui dresse des statues au comitium, qu'on sorte son portrait du temple de Jupiter Très Bon Très grand, même si, sur le tard, il fit preuve effectivement d'arrogance lorsqu'il dut faire face aux accusations de malversations portées contre son frère et luimême par ses adversaires politiques (Liv., XXXVIII, 56) ${ }^{39}$.

La mémoire des Scipions fut cependant défendue par le cercle des amis de Scipion Émilien. Polybe, qui en faisait partie, s'employa en particulier à démentir cette accusation d'affectatio regni, en évoquant précisément la façon dont le vainqueur de Bæcula avait refusé le titre royal que lui offraient les Ibères qu'il aurait invités à l'appeler "général " ${ }^{40}$. Ce passage, qui a pu inspirer le récit livien, est au reste d'une

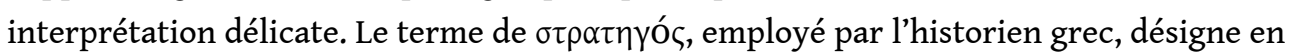
effet simplement le commandant en chef d'une armée, qu'il s'agisse d'un magistrat ou d'un pro-magistrat ${ }^{41}$; imperator, au sens de général victorieux, est quant à lui traduit

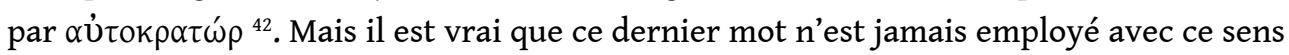
chez Polybe, mais avec celui de dictator, comme le fait justement remarquer E. Foulon,

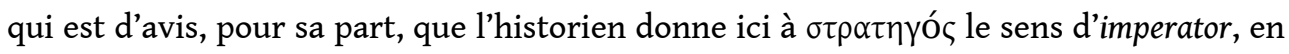
rapport avec l'idéologie de la victoire ${ }^{43}$. On remarquera cependant que c'est ce même titre de $\sigma \tau \rho \alpha \tau \eta \gamma o ́ \varsigma$ que les soldats puis le Conseil de Carthage attribuent à Hannibal lorsque celui-ci succède à Hasdrubal à la tête de l'armée d'Espagne, dans une position officielle très semblable à celle de Scipion; or le terme n'a dans ce passage de valeur qu'institutionnelle, et nullement la fonction honorifique d'imperator ${ }^{44}$. Il convient en outre de noter que, dans le cas de Scipion, Polybe ne fait aucune allusion au fait que celui-ci aurait été proclamé imperator par ses soldats, et qu'il invite les Ibères à l'appeler

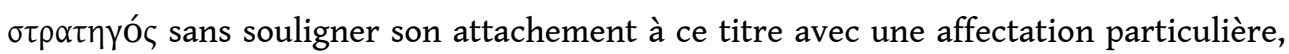
comme c'est le cas chez Tite-Live, ce qui semble indiquer que le mot n'a pas, lorsque 
Polybe l'emploie dans ce passage précis, de sens particulièrement fort. Le long commentaire de Polybe qui suit immédiatement (6-12) semble au demeurant vouloir accréditer la réalité de la modestie toute républicaine de Scipion dans cet épisode, en soulignant le paradoxe admirable que constituait aux yeux de l'historien l'attitude d'un homme dont les qualités et les exploits le rendaient digne d'être un roi comme le monde hellénistique en avait connu depuis Alexandre, mais qui «fit plus de cas de sa patrie et de la fidélité qu'il lui devait que de ce pouvoir vers lequel tout le monde dirige des regards d'envie admirative» (trad. D. Roussel). Polybe ne manque pas, au demeurant, de montrer combien ceux qui reconnaissent en lui des dons divins ou une proximité avec les forces divines se fourvoient et ne comprennent pas que le héros n'a jamais fait autre chose que de manifester la sûreté de son jugement, la grandeur de son esprit, la force de sa réflexion, son habileté de calculateur, ce qu'il ne manque pas d'applaudir ${ }^{45}$.

Il est donc intéressant de relever ici l'ambivalence fondamentale du personnage de Scipion chez Tite-Live. Celui-ci refuse de se faire le relais des accusations que, peut-être déjà Fabius Cunctator, en tout cas des hommes comme Caton, portèrent contre l'Africain, et reprend la version polybienne. Son héros doit être en effet emblématique des vertus qui ont permis à la République romaine de parvenir à son apogée morale et politique pendant la deuxième guerre punique. Mais il leur donne raison à long terme, puisque c'est le même Scipion qui, dans le récit livien, crée les conditions premières de l'évolution historique qui conduira à l'ère de ces imperatores qui prétendront gouverner le monde romain, et dont l'Africain est le prototype. On voit donc bien que le choix opéré par Tite-Live d'une version des faits où Scipion est présenté comme un imperator victorieux, et non pas comme un simple dux, n'est pas anodin, mais est en réalité riche en allusions et cohérent avec sa conception très catonienne de l'histoire ${ }^{46}$.

Il est encore un autre domaine où Tite-Live compare pour les différencier les comportements de Scipion et ceux d'Hannibal, c'est celui de la discipline militaire, que le général romain n'entend pas laisser dépérir comme l'a fait finalement Hannibal à Capoue. À plusieurs reprises, Tite-Live établit en effet un parallélisme entre les délices de Capoue, qui perdirent l'armée carthaginoise, et les délices de Syracuse, que Scipion sut éviter à son armée. C'est notamment le cas lors des attaques dirigées contre Scipion par Fabius Maximus, où le jeune général est accusé de "se comporter comme un roi étranger, aussi bien lorsqu'il ferme les yeux sur la licence des soldats que lorsqu'il les punit $"{ }^{47}$, dans le même temps qu'on lui reproche de se laisser aller à la séduction des délices de la grande cité sicilienne: «Il se promenait dans le gymnase vêtu d'un manteau et chaussé de sandales; il passait son temps à lire et à jouer à la palestre ; son état-major tout entier jouissait des agréments de Syracuse avec la même mollesse ; on avait oublié Carthage et Hannibal ${ }^{48}$; toute l'armée était corrompue par cette licence " 49. À ces accusations, le spectacle de la flotte en parfait ordre de marche que le jeune général est en mesure de montrer à la commission sénatoriale venue enquêter sur place constitue un démenti cinglant, qui marque toute la différence entre la simplicité des mœurs de Rome à ce moment de son histoire et la corruption de Carthage, engagée dans son déclin.

À la différence, également, d'un Hannibal à la tête d'une armée de mercenaires fidélisée à son chef, Scipion ne parvient pas, pour sa part, à imaginer que des soldats puissent faire dépendre leur loyauté et leur sens de la discipline de l'identité de leur chef, même si l'on sait que, par le passé, le même personnage a su habilement jouer de son charisme 
et de sa parenté avec les deux précédents généraux de l'armée pour établir des liens personnels avec ses troupes. Si cette dernière attitude préfigure narrativement celle des imperatores de la fin de la République, Scipion ne l'assume pas pour autant et son éthique reste encore complètement étrangère à ce type de relations faisant prévaloir la fides entre une armée et son général sur celle que cette dernière doit à la cité. Tel est le sens des reproches qu'il adresse à son armée stationnée à Sucro, après que celle-ci, croyant son général défunt, n'a pas hésité à se mutiner pour une question de solde en retard ${ }^{50}$. L'amplification du propos de Scipion, un long discours au style indirect, permet de souligner la gravité de ce comportement, qualifié au demeurant de portentum, comme s'il portait en lui une menace à long terme pour l'État ${ }^{51}$. On notera au reste l'absence significative chez Polybe, dans le même discours (XI, 28-29), de ce motif de la fragilité de la fidélité des troupes et de l'importance excessive accordée aux liens personnels avec le général.

On voit donc toute la complexité de l'écriture livienne qui parvient par ces structures narratives parallèles à marquer comment l'apparition d'un imperator tel que Scipion symbolise le début d'un nouveau cours de l'histoire romaine, tout en mettant en lumière, par des figures d'opposition, la différence entre l'évolution des deux cités rivales: si Carthage est engagée depuis longtemps dans l'ère des condottieri, Rome ne fait que s'y engager et est encore au sommet moral et politique de son évolution.

La volonté de mettre en parallèle les vies d'Hannibal et de Scipion est donc profondément ancrée dans le récit livien, même si l'idée n'en est pas nouvelle. En effet, suivant en cela l'exemple de Xénophon, Polybe, si ce n'est Fabius Pictor avant lui, n'avait pas résisté, à l'évidence, à la tentation de comparer les deux généraux qui avaient joué un rôle également déterminant, l'un en ouvrant la guerre, l'autre en y mettant un terme. Très vite, les idéologies politiques colorèrent les deux biographies : la tendance catonienne, peut-être dans une certaine continuité avec la ligne du Cunctator, hostile à l'hellénisation croissante de Rome, est sans doute à l'origine de cette ligne de partage entre, d'un côté, des aristocrates ambitieux, émules d'Alexandre, favorables à une expansion impériale outre-mer et soucieux de leur gloire, s'appuyant à la fois sur l'armée et le peuple (Hannibal, Scipion), de l'autre, leurs adversaires politiques, plus attentifs au bien public, rendant des comptes avant tout au Sénat, extrêmement méfiants à l'égard du monde grec, et prétendant préserver l'âme romaine en évitant sa contamination par le luxe de l'Orient hellénistique (Fabius Cunctator, Hannon, Caton). L'affirmation du conflit entre populares et optimates, à l'époque des Gracques, a contribué sans doute à "populariser» encore davantage ceux qui apparaissaient comme des personnages dont le poids politique avait pu inquiéter l'autorité sénatoriale, les rendant responsables des maux présents ou à venir de leur cité. Cette menace que Caton avait devinée derrière les grands généraux de Rome, pouvait aisément être reprise à son compte par un Tite-Live, témoin d'événements qui avaient finalement donné raison à l'ancien censeur. L'originalité livienne réside donc surtout dans une structuration des motifs biographiques permettant de mettre en perspective l'évolution historique de Rome, conformément à sa conception cyclique qui fait des années suivant la bataille du Métaure le point de départ d'une nouvelle tendance historique, du fait de l'affaiblissement du metus hostilis et du passage décisif des armées de Rome en Afrique et en Asie. Se profile alors dans le lointain l'ère des condottieri, émules d'Alexandre le Grand, qui devaient conduire la cité à sa perte, comme Hannibal avait contribué à le faire pour Carthage. 


\section{BIBLIOGRAPHIE}

CASSOLA F. 1962, I gruppi politici romani nel III secolo a. C., Trieste.

COMBÈs R. 1966, Imperator : recherches sur l'emploi et la signification du titre d'imperator dans la Rome républicaine, Publications de la Faculté des lettres et sciences humaines de l'Université de Montpellier 26, Paris.

DEININGER J. 1972, «Von der Republik zur Monarchie: die Ursprünge der Herrschertitulatur des Prinzipats », ANRW I, 1, p. 982-997.

DE SANCTIS G. 1936, « Imperator », in Studi in onore di Salvatore Riccobono, II, Palerme, p. 55-62.

DE SANCTIS G. 1970, Storia dei Romani. III, L'età delle guerre puniche, 1, Il pensiero storico 38, Florence

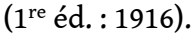

DUBUISSON M. 1985, Le latin de Polybe : les implications historiques d'un cas de bilinguisme, Études et commentaires 96 , Paris.

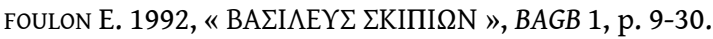

GAGÉ J. 1936, « De César à Auguste, où en est le problème des origines du principat ? ", RH 172, p. 64 .

GRIMAL G. 1975, Le siècle des Scipions : Rome et l'hellénisme au temps des guerres puniques, $2^{\mathrm{e}}$ éd. refondue et augmentée, Coll. historique, Paris.

MINEO B. 2006, Tite-Live et l'histoire de Rome, Études et commentaires 107, Paris.

NICOLET C. 1978, Rome et la conquête du monde méditerranéen, 264-27 avant J.-C. II, Genèse d'un empire, Nouvelle Clio 8 bis, Paris.

PICARD G.-C., PICARD C. 1970, Vie et mort de Carthage, Paris.

RICHARDSON J.S. 1991, « Imperium Romanum, Empire and the language of power », JRS 71, p. 1-9.

SCULLARD H.H. 1951, Roman Politics, 220-150 B.C., Oxford.

SCULLARD H.H. 1970, Scipio Africanus: Soldier and Politician, Aspects of Greek and Roman Life, Londres.

sYME R. 1979, « A Study in Nomenclature », in Id., Roman Papers, I, éd. E. Badian, Oxford, p. 361-377. TEDESCHI A. 1998, Lo storico in parola: Livio, Scipione l'Africano e le tecniche dell'argomentazione: commento a Liv. XXVIII, 43-44, Scrinia 13, Bari.

\section{NOTES}

1. Cornélius Népos (Hann. 13, 4) fait explicitement mention de son dessein de comparer la vie de généraux étrangers à celle des Romains de façon à établir des préférences.

2. Nep., Hann. 2, 4.

3. «L'historien romain Fabius Pictor nous dit que, parmi les causes de la guerre d'Hannibal, il y eut, outre l'agression contre Sagonte, l'ambition d'Hasdrubal et son amour du pouvoir. Ayant pris possession en Espagne d'un commandement important, celui-ci, selon notre auteur, se rendit en Afrique et entreprit d'abolir la constitution de Carthage pour lui substituer une forme de 
gouvernement monarchique... Il gouverna désormais selon son bon plaisir, sans tenir compte du Conseil de Carthage. Devenu, dès l'adolescence, le compagnon d'Hasdrubal et son émule, Hannibal, quand il eut succédé au gouvernement de l'Espagne, aurait continué sa politique. Ce serait la raison pour laquelle, de sa propre initiative, et passant outre l'avis des Carthaginois, il entreprit de faire la guerre aux Romains. Car parmi les notables de Carthage, il ne se trouvait personne, selon Fabius, pour approuver ce qu'Hannibal avait fait à Sagonte » (Plb., III, 8, 1-7, trad. D. Roussel). Polybe dénonce l'invraisemblance de la thèse de Fabius Pictor dans le passage qui suit immédiatement (III, 8, 9-11).

Selon Diodore (XXV, 8), Hamilcar, à force de démagogie, aurait poussé le peuple à lui laisser le commandement militaire pour une période indéterminée. Appien (Hann. I, 2 ; Hisp. 4-5) et Dion Cassius (XII, 17) font état d'une version selon laquelle Hamilcar aurait entrepris son expédition d'Espagne sans l'aval de Carthage. Sur la réalité historique d'un État barcide de facto indépendant, voir Picard, Picard 1970, p. 213 sq. Selon C. Nicolet, en revanche, les Barcides n'ont jamais rompu avec leur cité qu'ils ont servie avec dévouement, lors même qu'ils furent amenés par les circonstances à prendre des initiatives. Tout comme son père, Hannibal n'est rien d'autre qu'un général carthaginois, régulièrement nommé (Nicolet 1978, p. 566-567).

Selon F. Cassola, les Cornelii auraient fait leur priorité de l'expansion romaine en Méditerranée, rendant la confrontation avec Carthage inévitable; le Cunctator et ses alliés politiques, en revanche, auraient préféré consolider d'abord l'emprise de Rome sur la Gaule cisalpine. Fabius Pictor aurait alors présenté Hannon comme une sorte de pendant carthaginois du Cunctator qui se serait opposé, après la première guerre punique, à la politique d'expansion des Barcides en Espagne (De Sanctis 1970, p. 390). Ce clivage politique recoupait probablement celui mettant aux prises conservateurs hostiles à l'hellénisme (Fabius Maximus Cunctator, puis Caton) à l'aristocratie tentée par les séductions du monde hellénistique (Grimal 1975, p. 201-212). Par la suite, la carrière exceptionnelle de Scipion l'Africain, la façon dont celui-ci mit en échec la politique du Cunctator en 205, malgré son jeune âge, ont pu alimenter la crainte qu'inspiraient les généraux ambitieux qui regardaient vers l'Orient hellénistique, celle de voir des personnalités encombrantes, enorgueillies par les succès inouïs que leur avaient offerts la fortune (cela avait été le cas de Scipion à (arthagène), imposer leur autorité à l'État, tels de nouveaux Alexandre. Fabius Pictor peut-être déjà, puis très probablement Caton, ont aussi voulu illustrer cette menace-là, déjà dénoncée par Fabius Maximus. Et, pour appuyer leurs propos, ils auront présenté la situation à Carthage comme un miroir de celle qu'ils croyaient voir se dessiner à Rome.

Sur l'opposition des Fabii à un affrontement armé avec Carthage et à une aventure militaire en dehors de la péninsule italienne, et, à l'inverse, sur le rôle des Cornelii et de leurs alliés dans le déclenchement d'un conflit susceptible de favoriser le développement de la puissance romaine en Méditerranée, cf. Scullard 1951, p. 39-41, 61, 76 ; Cassola 1962, p. 259 sq.

4. Tite-Live ne reprend pas les affirmations de Fabius Pictor réfutées par Polybe, selon lesquelles Hannibal aurait engagé la guerre sans l'accord de Carthage. Le Padouan insiste au contraire sur la solitude d'Hannon au Sénat, face à l'ensemble de ses collègues, presque en totalité favorables à Hannibal (Liv., XXI, 11, 1).

5. Hamilcarem iunenem redditum sibi ueteres milites credere; eumdem uigorem in uoltu uimque in oculis, habitum oris lineamentaque intueri. Dein effecit ut pater in se minimum momentum ad fauorem conciiliandum esset (Liv., XXI, 4, 2, trad. CUF).

6. Liv., XXVI, 18, 7.

7. Vos modo, milites, nouum exercitum nouumque ducem traducite in terras cum multis fortibus factis scepe a uobis peragratas. Breui faciam ut, quemadmodum nunc noscitatis in me patris patruique similitudinem oris uoltusque et lineamenta corporis, ita ingenii fidei uirtutisque effigiem uobis reddam ut reuixisse aut renatum sibi quisque Scipionem imperatorem dicat (XXVI, 41, 22-25, trad. A. Flobert).

8. Liv., XXVIII, 40, 1-2. 
9. In Hasdrubalis locum haud dubia res fuit quinam sucessurus esset; prorogatiuam militarem qua extemplo iuuenis Hannibal in prctorium delatus imperatorque ingenti omnium clamore atque adsensu appellatus erat fauor etiam plebis sequebatur (Liv., XXI, 3, 1, trad. CUF).

10. Liv., XXV, 2, 7-8.

11. Liv., XXVI, 18, 5-10 (trad. B. Mineo).

12. Liv., $X L, 44,1-2$.

13. Liv., XXVII, 19.

14. Scipion, lorsqu'il traitera l'affaire des otages espagnols, établira des liens d'amitié avec Massinissa touché par la générosité dont le futur Africain témoignera à l'égard du jeune Massiva ; il sera aussi à deux doigts d'obtenir l'alliance avec Syphax, impressionné par le charisme du général romain, non moins que le général Hasdrubal, lui aussi présent à la rencontre.

15. Hasdrubal sut augmenter la puissance de Carthage en offrant l'hospitalité à de petits rois ou en gagnant, par l'amitié de leurs chefs, l'alliance de peuples nouveaux (Liv., XXI, 2, 5).

16. Selon Tite-Live, Scipion donnait du poids à ses propositions en laissant accroire qu'elles lui étaient inspirées par les dieux : il faisait notamment allusion à des apparitions nocturnes, à des avertissements divins, « soit parce qu'il était naturellement superstitieux, soit parce qu'il voulait qu'on admette sans discussion son point de vue ou son avis... il n'avait jamais pris de décision sans s'asseoir dans le temple et y demeurer un certain temps, généralement seul et à l'abri des regards... Il faisait ou laissait courir sur son compte une rumeur, selon laquelle il serait né fils d'un dieu, né d'un serpent monstrueux, récits qui rappelaient ceux qu'on avait inventés sur la naissance d'Alexandre le Grand. Il ne détruisit jamais ces croyances, il savait même les renforcer en se gardant à la fois de les démentir et de les confirmer... et c'est parce qu'ils y croyaient que ses concitoyens lui avaient confié si jeune une si lourde responsabilité et un commandement de cette importance » (Liv., XXVI, 19, 3-9, trad. A. Flobert). Scipion annonça aussi à ses troupes la future victoire en Espagne, en faisant état d'augures, d'auspices et de visions nocturnes (Liv., XXVI, 41). À propos de la marée qui facilite la prise de Carthagène, Tite-Live rapporte comment Scipion fit un miracle de ce qui n'était qu'un phénomène étudiable scientifiquement (Liv., XXVI, 45). De même avant Zama : «Il laissait entendre que les dieux avaient donné aux ennemis, au moment de partir au combat, les mêmes auspices qu'autrefois à leurs pères, avant la bataille des Îles Égates » (Liv., XXX, 32).

17. «De même Scipion, en faisant croire à ses troupes qu'une inspiration divine ne trouvait à l'origine de ses entreprises, obtint des hommes placés sous ses ordres qu'ils se lançassent dans des opérations périlleuses " $(\mathrm{Plb} ., \mathrm{X}, 2,12)$. De façon générale, il laissa accroire que ses décisions étaient inspirées par les dieux (Plb., X, 5, $5 ; \mathrm{X}, 11,7$ ), ce qui lui permettait d'arriver à ses fins. Polybe loue cette habileté à jouer de la crédulité des troupes (Plb., X, 5, 7-10). Sur la légende de Scipion, cf. Scullard 1970.

18. Liv., XXVIII, 40, 13-14 ; Tite-Live (XXX, 26) indique que Fabius mourut très âgé en 203 (donc peu de temps après ce débat), et qu'il avait été augure pendant 62 ans. Sur les qualités de uates qu'est susceptible de conférer l'extrême vieillesse, cf. Cic., Diu. I, 63-64.

19. L'intervention d'Hannon au début de la deuxième guerre punique est du reste d'autant plus étrange que Tite-Live s'évertue à mettre en scène l'adversaire des Barcides à chacune des grandes étapes du conflit, après Cannes, et même au moment du rappel d'Hannibal d'Italie (Liv., XXIII, 12 ; $\mathrm{XXX}, 20)$. On notera du reste que ce personnage est absent du texte de Polybe traitant de ces événements, ainsi que de celui de Diodore de Sicile.

20. Hannon dénoncera du reste de nouveau le désir de régner d'Hannibal à l'occasion de son autre intervention au Sénat, lors de l'arrivée de l'ambassade romaine à Carthage : "C'est un jeune homme brûlant du désir de régner (iuuenem flagrantem cupidine regni), aux yeux duquel le seul moyen d'y parvenir était de vivre entouré d'armes et de légions en semant guerre sur guerre, que vous avez envoyé aux armées, comme on jette du bois pour alimenter le feu » (Liv., XXI, 10, 4). Ces craintes se vérifieront par la suite par le peu de cas qu'Hannibal fera du Sénat au 
moment d'engager son expédition en Italie, et surtout dans cette fameuse scène, dont on retrouve l'évocation chez Polybe (Plb., XV, 19, 3), où, de retour à Carthage, après Zama, il prend violemment à parti un orateur qui s'opposait à ses vues : "Cette attitude, déplacée dans un régime démocratique, provoqua la colère de l'assistance ; sincèrement surpris de la liberté qui régnait dans la ville, en homme habitué à la discipline militaire, Hannibal répliqua : "Parti à neuf ans, je reviens après trente-six ans d'absence; je crois bien connaître la vie militaire à laquelle j'ai été formé dès l'enfance, par tradition familiale puis par les charges que j'ai remplies ; mais il faudra que vous m'appreniez les droits, les institutions et les usages qui règlent la ville et le forum." " (Liv., XXX, 37, trad. A. Flobert).

21. Liv., XXVIII, 42, 22 (trad. A. Flobert modifiée).

22. L'importance du modèle thucydidéen a été perçue par Tedeschi 1998, p. 90-91, qui relève surtout dans son analyse du débat entre Fabius et Scipion la place du conflit entre générations.

23. Th., VI, 13, 1 : « Pour ma part, quand je vois cette jeunesse siéger ici aujourd'hui sur l'appel de ce même personnage, je m'effraie; et je fais en revanche appel aux hommes d'âge...» (trad. J. de Romilly).

24. Th., VI, 17, 1.

25. Liv., XXV, 2, 7-8; XXVI, 18, 5-10.

26. Th., VI, $15,5$.

27. Liv., XXIX, 19, 11-13.

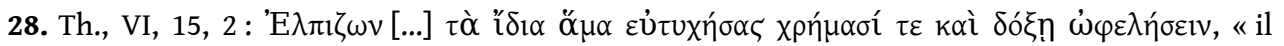
espérait [...] si la fortune le favorisait, de servir ses intérêts privés sous le rapport de l'argent et de la réputation » (trad. J. de Romilly) ; Nicias $(13,2)$, quant à lui, en appelle à l'intérêt de la patrie (ن் $\tilde{\eta} \rho \tau \tilde{\eta} \varsigma ~ \pi \alpha \tau \rho i ́ \delta o \zeta)$. À comparer avec les reproches adressés par Fabius (Liv., XXVIII, 42, 22) à Scipion: Ego, patres conscripti, P. Cornelium rei publicee nobisque, non sibi ipsi priuatim creatum consulem existimo.

29. Th., VI, 13, 2 (trad. J. de Romilly).

30. Th., VI, 15, 5 ; Liv., XXVIII, 42, 22.

31. Liv., XXVIII, 41, 17. La tradition sur laquelle s'appuie Tite-Live est pratiquement impossible à définir, faute d'avoir conservé les passages éventuellement correspondants de Fabius Pictor, de Polybe ou de Caton. Il est possible que le Cunctator se soit effectivement opposé à Scipion en 205 pour des raisons de stratégie, de rivalité politique, de méfiance à l'égard de Scipion (Plutarque explique son hostilité au plan de Scipion par de la jalousie) ; mais il n'est pas certain que la prise de position idéologique (les dangers d'un impérialisme aventureux) ne doivent pas beaucoup avant tout aux Origines de Caton.

32. Mineo 2006, p. 293-322.

33. La réalité de cet enjeu est mise à jour par Hannibal lui-même, lors de sa rencontre avec Scipion avant la bataille de Zama. Chargé d'expérience, le Carthaginois a su apprécier la sagesse de ceux qui, comme Hannon ou Fabius, avaient préconisé de ne pas s'aventurer au-delà de la sphère naturelle d'influence des deux puissances : « Le mieux eût été que les dieux aient donné à nos ancêtres l'intention de se contenter d'une domination sur l'Italie, pour ce qui vous concerne, sur l'Afrique, pour ce qui est de nous " (Liv., XXX, 30, 6, trad. B. Mineo). Une leçon de l'histoire que le même personnage avait déjà énoncée dans la version polybienne. Hannibal regrette aussi la foi excessive qu'il mettait en sa fortune personnelle (Liv., XXX, 30, 18), attitude qui est celle du héros hellénistique que lui-même et Scipion incarnent dans le récit. Il ne manque pas de souligner le parallèle sur ce point entre lui-même et Scipion, qui est dans le présent ce qu'Hannibal était à Trasimène et à Cannes (Liv., XXX, 30,12). Et de rappeler l'inutilité de l'exaltation guerrière du héros, encouragé par ses grandes victoires, quand on connaît la capacité de la fortune à renverser les situations les mieux établies (Liv., XXX, 30, 15). L'ensemble de ces déclarations sont du reste dans la logique de la tradition polybienne, qui semble avoir eu à cœur de comparer le destin des deux condottieri. 
La réaction de Scipion au discours d'Hannibal témoigne quant à elle de l'arrogance qu'inspire au futur vainqueur de Zama sa jeunesse et sa confiance en sa fortune $(30,31)$. Son aveuglement est manifeste et nécessaire pour que s'effectue le changement d'échelle historique et le destin de Rome dont il est responsable dans le récit livien.

34. Liv., XXXVIII, 50 (trad. A. Flobert).

35. Les propos du frère de Scipion, Lucius, lors de son procès intervenant après la mort de Publius, reprennent le thème de l'inuidia dont Scipion comme Hannibal furent les victimes : «Le départ d'Hannibal pour l'exil avait suffi aux Carthaginois, même la mort n'avait pas désarmé la colère des Romains contre P. Scipion » (Liv., XXXVIII, 54). L'ingratitude de Rome à son égard a pu être réellement ressentie par l'Africain. Tite-Live rappelle qu'il ne voulut pas de funérailles dans sa patrie ingrate (XXXVIII, 53). Valère Maxime nous a conservé l'épitaphe amère qui aurait été composée à l'occasion par Ennius : Ingrata patria, ne mea quidem ossa habeas, "ingrate patrie, tu n'auras pas même mes os » (Val. Max., V, 3, 2).

36. La rencontre entre Scipion et Hannibal à Éphèse, peut-être inventée par Acilius, et rapportée par Claudius Quadrigarius, selon Tite-Live (Liv., XXXV, 14), devait correspondre à cette habitude de comparer les talents des généraux. Dans cette joute comparative, on sait qu'Hannibal devait déclarer Scipion « hors concours ».

37. Liv., préf. 9.

38. Liv., XXVII, 19 (trad. B. Mineo). Sur les visées monarchiques dont fut parfois soupçonné Scipion, cf. Foulon 1992. H.H. Scullard (1951, p. 84) envisage que le refus de la monarchie prêté au vainqueur de Zama ne reflète en réalité le peu d'intérêt marqué par Scipion Émilien après la destruction de Carthage pour devenir roi.

39. Cette information, que Tite-Live dit avoir trouvée dans un discours de Ti.Sempronius Gracchus, serait en réalité l'élément d'un pamphlet dénonçant le comportement politique de Sylla ou de César (Scullard 1951, p. 84).

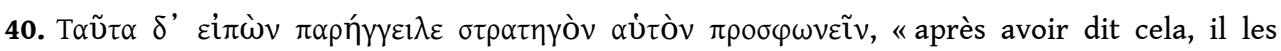
engagea à l'appeler « général » (Pol., X, 40,5).

41. Dubuisson 1985, p. 45-47.

42. D. H., IX, 3, 4 ; App., B. C. II, 178. Sur imperator, cf. Combès 1966 ; Deininger 1972 ; Richardson 1991, p. 1-9.

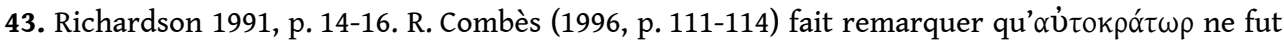
employé avec le sens honorifique d'imperator qu'à partir de Sylla $(\mathrm{p} .56 ; 11-114)$ et qu'il était

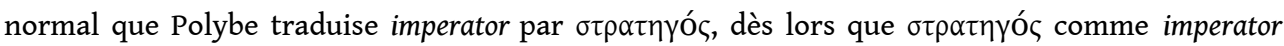
désignaient des chefs d'armée (p. 56) ; quant au fait que Polybe ne commente pas la nouveauté de la proclamation, cela s'expliquerait simplement par la préoccupation de l'historien de souligner la grandeur du refus de Scipion (loc. cit.). M. Dubuisson (1985, p. 47) fait aussi de $\sigma \tau \rho \alpha \tau \eta \gamma o ́ \varsigma$ l'équivalent du titre honorifique d'imperator. En revanche, G. De Sanctis (1936, p. 58) est d'avis que le titre d'imperator servait en temps normal aux soldats pour s'adresser à leur chef, sans que ce titre eût une valeur officielle.

44. Plb., III, 13, 4 .

45. Plb., X, 2, 4-5.

46. Il est intéressant de relever que le refus opposé par Scipion est sans appel et contraste avec l'ambiguïté de l'attitude de César qui, dans une scène que Tite-Live pouvait avoir à l'esprit en écrivant ce passage, décline le titre de roi que lui décernent certains de ses partisans tandis qu'il fait son entrée dans la ville, conscient de l'impopularité suscitée par cette initiative (César aux portes de Rome : Appien, B. C. II, 108 ; le refus de César lors des Lupercales : ibid., 109). Le refus du diadème par le dictateur, lors des Lupercales, obéit à la même logique. La mise en scène livienne nous rappelle en réalité deux préoccupations essentielles pour Octave au moment où celui-ci construisait les fondements idéologiques de son pouvoir. Il ne fallait pas prêter le flanc, comme son divin père, aux accusations d'affectatio regni. L'on sait, par ailleurs, combien il lui tint à cœur 
d'afficher son titre d'imperator : à partir de 38 au moins, ce titre remplaçait du reste son pronomen , comme l'attestent les monnaies frappées en Gaule par Agrippa à cette date (BMC, R. Rep. II, 411 ff. Gagé 1936, p. 64). Pendant longtemps, jusqu'au vote des honneurs du 16 janvier 27, ce seul titre fut presque toute la gloire du personnage, si l'on veut bien ne pas tenir compte du prestige que lui valait son nom d'adoption et sa qualité de Diui filius (sur la titulature d'Auguste, cf. Syme 1979).

47. Liv., XXIX, 19, 4: Externo et regio more et indulgere licentice militum et sceuire in eos (trad. B. Mineo).

48. Liv., XXIX, 19, 12-13: Cum pallio crepidisque inambulare in gymnasio; libellis eum palcestrceque operam dare; æque molliter cohortem suam totam Syracusarum amœnitate frui; Carthaginem atque Hannibalem excedisse de memoria ; exercitum omnem licentia corrumptum (trad. B. Mineo).

49. Lors du procès contre les Scipions (XXXVIII, 51, 1), les tribuns de la plèbe ne se feront pas faute de rappeler les uetera luxurice crimina Syracusanorum hibernorum. Enfin, après Zama, Hannibal lui-même opposera son attitude après Cannes (implicitement les délices de Capoue) à celle de Scipion qui fut bien avisé de marcher contre Carthage et maudissait « l'erreur qu'il avait commise en ne conduisant pas devant Rome ses soldats encore tout sanglants de la victoire de Cannes : Scipion, qui n'avait pas vu l'ennemi carthaginois en Italie quand il était consul, avait osé marcher sur Carthage » (Liv., XXX, 20).

50. Liv., XXVIII, 28, 10, 11 : «Et quoi ? Si j'étais mort, l'État devrait-il périr avec moi, est-ce que l'empire du peuple romain devrait tomber en même temps que moi ? Que Jupiter Très Bon Très Grand ne permette pas qu'une ville fondée après la consultation des auspices et avec la garantie des dieux pour l'éternité ne puisse durer davantage que mon corps fragile et mortel» (trad. B. Mineo).

51. Liv., XXVIII, 27, 16: «Il s'agit là d'un prodige qui ne saurait être conjuré sans victimes, sans supplications, sans verser le sang de ceux qui ont eu l'audace d'un crime si grand» (trad. B. Mineo).

\section{RÉSUMÉS}

Le propos de cet exposé est d'illustrer la réalité de ce projet de vies parallèles et d'en éclairer la raison d'être. À cette fin, on examine tour à tour les motifs qui permettent à l'auteur de souligner les analogies entre les deux héros, de dégager ensuite les structures narratives porteuses de ces parallélismes biographiques ainsi que les procédés rhétoriques les mettant en valeur (amplification, dispositio) ; enfin, on fait apparaître les éléments de différenciation entre les deux vies susceptibles d'éclairer le sens de la construction livienne. 


\section{INDEX}

Mots-clés : avaritia, biographie, dispositio, guerres puniques, héroïsme, historiographie hellénistique, historiographie romaine, idéologie dynastique, impérialisme antique, intertextualité, luxuria, morale et littérature, portrait, psychologie littéraire, royauté à Rome, Sénat romain, vie (genre littéraire)

nomsmotscles Appien, Cicéron, Diodore de Sicile, Dion Cassius, Ennius, Fabius Pictor, Nepos (Cornelius), Polybe, Rutilius Rufus, Tite-Live, Thucydide, Varron, Xénophon Index géographique : Afrique, Cannes, Capoue, Carthage, Corse, Espagne, Métaure, Sardaigne, Sicile, Sucro, Zama

Keywords : avaritia, biography, dispositio, Punic wars, heroism, hellenistic historiography, Roman historiography, dynastic ideology, ancient imperialism, intertextuality, luxuria, ethics and literature, portrait, literary psychology, Roman kingship, Roman senate, life (literary genre) 